\title{
On simplicial and central measures, and split faces.
}

By

Asvald Lima

The main purpose of this paper is to study the facial structure of compact convex sets. The results we obtain are used to give characterizations of simplicial and central measures.

In the first two sections we restrict our attention to the compact convex set of all probability measures on a compact convex set. In section 1 it is shown that a probability measure is a simplicial measure if and only if the restriction of the barycenter map to the smallest face containing the measure is injective.

It is well known that a compact convex set is a Choquet simplex if and only if the restriction of the barycenter map to the set of all maximal probability measures is injective. This is used in section 2 to show that the norm closure of a face in a compact Choquet simplex is a face.

Section 3 contains some results on strongly disjointness and split faces.

The last section is about contral measures. We give characterizations of central measures and we show that every central measure is a simplicial measure. 
Notation

Let $K$ be a compact convex set in a real locally convex Hausdorff vector space $E$. $A(K)$ will denote the (real) affine continuous functions on $K$ and $C(K)$ the space of all (real) continuous functions on $K$. Without loss of generality we can assume that $E=I$ in span $K$ and that $K \subseteq H$ where $H$ is a hyperplane in $\mathrm{E}$ and $\mathrm{O} \notin \mathrm{H}$. (See Theorem II.2.4. in [3].)

If $X$ is a Borel subset of $K$, we shall denote by $M(X)$ the space of all signed Borel measures on $K$ such that $|\mu|(K \backslash X)=0$, and by $\mathbb{M}_{1}^{+}(X)$ the convex set of normalized positive measures in $M(X)$. If $X$ is compact, $M(X)$ is a Banach space and $\mathbb{M}_{1}^{+}(X)$ is $W^{*}$-compact. (We will identity $M(X)$ and $C(X) *$ when $X$ is compact.)

A signed measure $\mu$ on $K$ is said to be maximal or a boundary measure if $|\mu|$ is maximal in Choquet's ordering, $<$, of the positive measures $\mathrm{M}^{+}(\mathrm{K})$. The linear subspace of $\mathbb{M}(K)$ of alI (signed) boundary measures is denoted by $Q$ and $Q_{1}=Q \cap M_{1}^{+}(K)$. If $\mu \in \mathbb{M}_{1}^{+}(K)$, then the barycenter or resultant of $\mu$ is the unique point $\mathrm{x}=r(u) \in K$ such that $\int_{\mathrm{K}} \mathrm{f} a \mu=f(x)$ for all $f \in A(K)$. The map $r: \mathbb{M}_{1}^{+}(K) \rightarrow K$ defined by $\mu \rightarrow r(\mu)$ is continuous, surjective and affine and the restriction of $r$ to the face $Q_{1}$ is also surjective. (See [3] or [15].) We shall write $\mathbb{M}_{X}$ for the set $\left\{\mu \in \mathbb{M}_{1}^{+}(K): r(\mu)=x\right\}$ and $Q_{x}$ for $M_{X} \cap Q_{1}$, $\mathrm{x} \in \mathrm{K}$.

If $S \subseteq K$, then $c o(S)$ is the convex hull of $S$, and $\overline{c o}(S)$ is the closed convex hull of $S$. If $C$ is a convex set, $\partial_{e} \mathrm{C}$ will denote the set of extreme points in $\mathrm{C}$ (if any). The reader is refered to [3] as a general reference on compact convex sets. 
1. Characterizations of simplicial neasures

Definition: If $S \subseteq K$, the smallest face in $K$ containing $S$ will be denoted by face (S) .

The following characterization of face $(x), x \in K$, is well known (see e.g. [1]).

Proposition 1.1: Let $\mathrm{x} \in \mathrm{K}$.

$$
\text { face } \begin{aligned}
(x) & =\bigcup_{\alpha \geq 1}(\alpha x-(\alpha-1) K) \cap K \\
& =\left\{y \in K: x=\frac{1}{\alpha} y+\left(1-\frac{1}{\alpha}\right) z \text { for some } z \in K \text { and } \alpha \geq 1\right\}
\end{aligned}
$$

Proposition 1.2: Let $\mu \in \mathbb{M}_{1}^{+}(\mathrm{K})$. Then we have:

face $(\mu)=\left\{\nu \in \mathbb{M}_{1}^{+}(K): \nu<<\mu\right.$ and $\left.\frac{d \nu}{d \mu} \in I^{\infty}(\mu)\right\}$

Proof: Let $\nu \in$ face $(\mu)$. Then by Proposition 1.1 there exist $\pi \in \mathbb{M}_{1}^{+}(K)$ and $\left.\alpha \in<0,1\right]$ such that

$$
\mu=\alpha \nu+(1-\alpha) \pi
$$

If $B \subseteq K$ is a Borel set, then $\mu(B) \geq \alpha \nu(B)$, and hence $\nu \ll \mu$ with $\frac{d \nu}{d \mu} \in I^{\infty}(\mu)$.

Suppose next that $\nu \in \mathbb{M}_{1}^{+}(K)$ and $\nu \ll \mu$ with $\frac{d \nu}{d \mu} \in I^{\infty}(\mu)$. Let $\alpha=1+\left\|\frac{d \nu}{d \mu}\right\|_{I} \longrightarrow(\mu)$.

Define a measure $\pi$ by

$$
\pi(B)=(\alpha-1)^{-1}(\alpha \mu(B)-\nu(B))
$$

for each Borel subset $B$ of $K$. Then $\pi \in \mathbb{M}_{1}^{+}(K)$ and $\mu=\frac{1}{\alpha} \nu+\left(1-\frac{1}{\alpha}\right) \pi$, such that $\nu \in$ face $(\mu)$. The proof is complete. 
Proposition 1.3: Let $\mu \in \mathbb{M}_{1}^{+}(\mathrm{K})$. Then we have $\overline{\text { face }(\mu)^{\text {norm }}}=\left\{\nu \in \mathbb{M}_{1}^{+}(K): \nu \ll \mu\right\}$

Proof: 1) Suppose $\nu \in \mathbb{M}_{1}^{+}(K)$ and $\nu \ll \mu$. Let $f=\frac{d \nu}{d \mu} \in I^{1}(\mu)$. For each $n \in\{1,2, \ldots\}$, define

$$
f_{n}=(\mu(\min (n, f)))^{-1} \cdot \min (n, f),
$$

and define a measure $\mu_{n}$ by

$$
\mu_{n}(B)=\int_{B} f_{n} d \mu
$$

for each Borel set $B \subseteq K$. Then $\mu_{n} \in$ face $(\mu) . \quad 1=\left\|f_{n}\right\|_{I^{1}(\mu)}=$ $\|f\|_{L^{1}(\mu)}$ and $f_{n}(x) \rightarrow f(x)$ a.e. $(\mu)$, so $f_{n} \rightarrow f$ in $I^{1}(\mu)$. Thus we have:

$$
\begin{aligned}
& \left\|\nu-\mu_{n}\right\|=\sup _{\substack{\|h\| \leq 1 \\
h \in C(K)}}\left|\nu(h)-\mu_{n}(h)\right| \\
= & \sup _{\|h\| \leq 1}\left|\int h\left(f-f_{n}\right) d \mu\right| \leq\left\|f-f_{n}\right\|_{L^{1}(\mu)} \rightarrow 0 \text {, as } n \rightarrow \infty . \\
h \in C(K) &
\end{aligned}
$$

Hence $\nu \in \overline{\text { face }(\mu)}^{\text {norm. }}$

2) Suppose next that $\nu \in{\overline{\text { face }(\mu)^{\text {norm. }}}}^{\text {For each Borel }}$ subset $A$ of $K$ such that $\mu(A)=0$, we define a face $F$ by

$$
\mathrm{F}=\left\{\sigma \in \mathbb{M}_{1}^{+}(\mathrm{K}): \sigma(\mathrm{A})=0\right\}
$$

Using regularity of Borel measures and Urysohn's lemma, we see that $F$ is norm-closed.

Thus

$F=\left\{\sigma \in \mathbb{M}_{1}^{+}(K): \sigma(A)=0\right.$ for each Borel set in $K$ with $\mu(A)=0\}$

is a norm-closed face. Clearly $\mu \in F$, and $\sigma \in F$ if and only

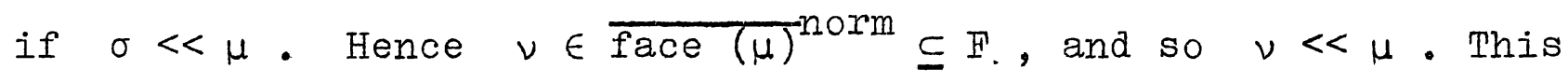
completes the proof. 
Corollary 1.4: Let $\mu \in \mathbb{M}_{1}^{+}(\mathrm{K})$. Then $\overline{\text { face }(\mu)^{\text {norm }}}$ is a face.

It is well known that the $w^{*}-c l o s u r e$ of a face of the Bauer simplex $\mathbb{M}_{1}^{+}(K)$ must be a face [1]. By Corollary 1.4. it follows that the corresponding statement is valid for the norm closure of face $(\mu)$. In Theorem 2.5. we shall prove that the norm closure of any face in $\mathbb{M}_{1}^{+}(K)$ is a face and in Corollary 2.7. we shall prove that the norm closure of any face in a compact simplex is a face.

Definition: Let $x \in K$ and $\mu \in \mathbb{M}_{X}$. We shall say that $\mu$ is simplicial if $\mu$ is an extreme point in $M_{x}$.

If $x \in K$ and $\mu \in \mathbb{M}_{x}$, we have that $r^{-1}($ face $(x))$ is a face in $\mathbb{M}_{1}^{+}(K)$ containing $\mu$. Thus face $(\mu) \subseteq r^{-1}$ (face $(x)$ ), and hence $r($ face $(u)) \subseteq$ face $(x)$.

In the following theorem (i) $\Longleftrightarrow$ (ii) was first proved by Douglas $[7]$.

Theorem 1.5: Let $x \in \mathbb{K}$ and $\mu \in \mathbb{M}_{X}$. The following statements are equivalent:

(i) $\mu$ is a simplicial measure.

(ii) $A(K)$ is dense in $I^{1}(\mu)$.

(iii) The restriction of $r$ to face ( $\mu$ ) is injective.

(iv) For every $\nu \in \mathbb{M}_{X}$ such that $r($ face $(\nu)) \subseteq r($ face $(\mu))$, we have $\nu<\mu$.

(v) The set $\left\{\nu \in \mathbb{M}_{x}: \nu<\mu\right\}$ is a face in $\mathbb{M}_{x}$. 
Proof: (i) $\Longleftrightarrow$ (ii) see [7] or [3].

(ii) $\Longrightarrow$ (iii) Suppose $\nu_{1}, \nu_{2} \in$ face $(\mu)$ and $r\left(\nu_{1}\right)=r\left(\nu_{2}\right)$. Let $f_{1}, f_{2} \in I^{\infty}(\mu)$ be such that $\nu_{1}=f_{1} \cdot \mu$ and $\nu_{2}=f_{2} \cdot \nu$. For every $a \in A(K)$ we have

$$
a\left(r\left(\nu_{1}\right)\right)=\int a d \nu_{1}=\int a f_{1} d \mu
$$

and

$$
a\left(r\left(\nu_{2}\right)\right)=\int a d \nu_{2}=\int a f_{2} d \mu
$$

such that

$$
0=\int a\left(f_{1}-f_{2}\right) d \mu
$$

Since $A(K)$ is dense in $I^{1}(\mu)$, we have $f_{1}=f_{2}$ a.e. $(\mu)$, and hence $\nu_{1}=\nu_{2}$.

(iii) $\Longrightarrow$ (ii) Suppose $r$ restricted to face $(\mu)$ is injective and that $A(K)$ is not dense in $I^{1}(\mu)$. Then there exists a $\varphi \in I^{\infty}(\mu)$ such that $\varphi \neq 0$ and $\int a \varphi d \mu=0$ for all $a \in A(K)$. Now we have $\varphi=\varphi_{1}-\varphi_{2}$ where $\varphi_{1}, \varphi_{2} \geq 0$, and we can suppose that $1=$ $\int \rho_{1} d \mu=\int \varphi_{2} d \mu$. Define measures $\nu_{1}$ and $\nu_{2}$ by

and

$$
\nu_{1}=\varphi_{1} \cdot \mu
$$

$$
\nu_{2}=\varphi_{2} \cdot \mu
$$

Then $\nu_{1}, \nu_{2} \in$ face $(\mu)$ and $r\left(\nu_{1}\right)=r\left(\nu_{2}\right)$ even though $\nu_{1} \neq \nu_{2}$. Thus we have a contradiction.

(iii) $\Longrightarrow$ (iv) Suppose $\nu \in \mathbb{M}_{X}$ and $r($ face $(\nu)) \subseteq r($ face $(\mu))$. Suppose $\nu=\sum_{i=1}^{n} \lambda_{i} \nu_{i}$ where $\nu_{i} \in \mathbb{M}_{1}^{+}(K), \lambda_{i}>0$ and $\sum_{i=1}^{n} \lambda_{i}=1$. Each $\nu_{i} \in$ face $(\nu)$, so $r\left(\nu_{i}\right) \in r($ face $(\mu)), i=1, \ldots, n$. Thus we can find a (unique) $\mu_{i} \in$ face $(\mu)$ such that $r\left(\nu_{i}\right)=r\left(\mu_{i}\right)$, $i=1, \ldots, n$. Now $r\left(\sum_{i=1}^{n} \lambda_{i} \mu_{i}\right)=\sum_{i=1}^{n} \lambda_{i} r\left(u_{i}\right)=\sum_{i=1}^{n} \lambda_{i} r\left(\nu_{i}\right)=$ $r(\nu)=x=r(\mu)$. Since $\mu_{i} \in$ face $(\mu), i=1, \ldots, n$, and the restriction of $r$ to face $(\mu)$ is injective, we have $\mu=\sum_{i=1}^{n} \lambda_{i} \mu_{i}$. From Corollary I.3.3. in [3] it follows that $\nu<\mu$. 
(iv) $\Longrightarrow(\mathrm{v})$ Suppose $\nu_{1}, \nu_{2} \in \mathbb{M}_{1}^{+}(\mathrm{K}), \lambda \in<0,1>$ and $\nu=\lambda \nu_{1}+$ $(1-\lambda) \nu_{2}<\mu$. Obviously we have face $\left(\nu_{i}\right) \subseteq$ face $(\nu)$ and $r\left(\right.$ face $\left.\left(\nu_{i}\right)\right) \subseteq r($ face $(\nu)), i=1,2$. Suppose $y \in r($ face $(\nu))$. Then there exist $a \in<0,1]$ and $\pi_{1}, \pi_{2} \in$ face $(\nu)$ such that $r\left(\pi_{1}\right)=y$ and

$$
\nu=x \pi_{1}+(1-\alpha) \pi_{2}
$$

Since $\nu<\mu$, there exist (by Corollary I.33. in [3]) $\mu_{1}, \mu_{2} \epsilon$ face $(\mu)$ such that $r\left(u_{i}\right)=r\left(\pi_{i}\right), i=1,2$, and

$$
u=\alpha u_{1}+(1-\alpha) \mu_{2} \text {. }
$$

Thus we have $y \in r($ face $(\mu))$. Hence

$$
r\left(\text { face }\left(\nu_{i}\right)\right) \subseteq r(\text { face }(\nu)) \subseteq r(\text { face }(\mu)) \quad, i=1,2 .
$$

Now it follows from (iv) that if $\nu_{1}, \nu_{2} \in \mathbb{M}_{x}$, then $\nu_{1}, \nu_{2}<u$. $(v) \Longrightarrow(i)$. Suppose $\mu_{1}, u_{2} \in \mathbb{M}_{x}$ and $\mu=\frac{1}{2} \mu_{1}+\frac{1}{2} u_{2}$. Then $\mu_{1}, u_{2}<\mu$. Hence $\mu_{1}(f)=u_{2}(f)=\mu(f)$ for all convex functions in $C(K)$. Hence $\mu_{1}=\mu_{2}=u$. The proof is complete.

Remark: The argument used to prove (iii) $\Rightarrow$ (iv) in Theorem 1.5. was also used by Wils in [22]. A related result observed by Wils [22], Lemma 3.19.] is the following proposition.

Proposition 1.6: Let $\mu, \nu \in \mathbb{M}_{x}$. If $\nu<\mu$, then $r($ face $(\nu)) \subseteq r($ face $(\mu))$.

Proof: See the proof of (iv) $\Rightarrow(\mathrm{v})$ in Theorem 1.5 .

Corollary 1.7: Let $\mu \in \mathbb{M}_{\mathrm{X}}$ be simplicial. Then $r$ (face $(u)$ ) = face $(x)$ if and only if $\mu$ is the unique maximal measure in $\mathbb{M}_{x}$. 
Proof: Follows from Theorem 1.5. and Proposition 1.6.

Remark. Let $u \in \mathbb{M}_{1}^{+}(K)$ and define $B_{\mu}=\left\{f \in I^{\infty}(\mu): f \geq 0\right.$ and $\left.\int f d u=1\right\} . B_{u}$ is a basis for the positive cone in $I^{\infty}(u)$, and we can define a map $\varphi_{\mu} \dot{B}_{\mu} \rightarrow K$ by

$$
\psi_{\mu}(f)=r(f \cdot \mu) \text {. }
$$

$\varphi_{\mu}$ is affine and $\varphi_{u}$ has a unique extension to a linear functional $\Phi_{\mu}: I^{\infty}(\mu) \rightarrow E$. This map $\Phi_{\mu}$ plays a central role in [22].

Proposition 1.8: Let $x \in K$. Consider the following statements:

(i) $Q_{X}$ consists of a unique measure.

(ii) For all $y, z \in K$ and $\lambda \in[0,1]$ such that $x=\lambda y+(1-\lambda) z$, $Q_{x}=\lambda Q_{y}+(1-\lambda) Q_{z}$.

(iii) $\hat{f}$ face $(x)$ is affine for every convex function $f \in C(K)$.

Proof: (i) $\Rightarrow$ (ii) is trivial.

(ii) $\Longrightarrow$ (i) By Prop.I.6.12. in [3], there exists a simplicial $\mu \in Q_{X}$. Obviously by (ii), we have $r$ (face $\left.(\mu)\right)=$ face $(x)$. From Corollary 1.7. we can conclude that $Q_{X}=\{\mu\}$.

(i) $\Rightarrow$ (iii). Let $f \in C(K)$ be a convex function. If $y=$ face( $(x)$, then it follows from (i) that $Q_{y}$ consists of a unique measure. Now (iii) follows from Prop.I.6.12. in [3]. (iii) $\Rightarrow$ (i). Let $\mu \in Q_{X}$. Let $\left\{\mu_{a}\right\} \subseteq \mathbb{M}_{X}$ be a net of discrete measures such that $\mu_{\alpha}<\mu$ for all $\alpha$ and $\mu=\lim _{\alpha} \mu_{\alpha}$ (w*-Iimit). (See [3, Prop.I.2.3.].) Let $f \in C(K)$ be a convex function. By Prop.I.3.5. in [3], to each $\mu_{\alpha}$, we can find a $\nu_{\alpha} \in \mathbb{M}_{x}$ such that $u_{\alpha}<\nu_{\alpha}$ and $\nu_{\alpha}(f)=\mu_{\alpha}(\hat{f})$. Going to a subnet if necessary, we can assume $\nu_{\alpha} \rightarrow \nu\left(w^{*}\right)$ for some $\nu \in \mathbb{M}_{X}$. 
If $g \in C(K)$ is a conves function, then

$$
\nu(g)=\lim _{\alpha} \nu_{\alpha}(g) \geq \lim _{\alpha} \mu_{\alpha}(g)=\mu(g) .
$$

Hence $u<v$. Since $\mu$ is a maximal we have $\mu=\nu$. Thus $\mu(f)=\lim _{\alpha} \nu_{\alpha}(f)=\lim _{\alpha} \mu_{\alpha}(\hat{f})$. If $\hat{f}$ is affine on face $(x)$, then since $\mu_{\alpha}$ is discrete, $\mu_{\alpha}(\hat{f})=\hat{f}(x)$. Thus $\mu(f)=\hat{f}(x)$. If $\sigma \in Q_{X}$, then

$\mu(f)=\hat{f}(x)=\sigma(f)$, all convex $f \in C(K)$.

Hence $\mu=\sigma$, and $Q_{X}$ consists of a unique measure. The proof is complete.

Remark: In the above proposition (i) $\Rightarrow$ (iii) was first proved by Køhn in [11]. He left it as an open problem whether (iii) $\Rightarrow$ (i). 


\section{Characterization of split faces.}

Before stating the theorem characterizing split faces, we shall establish some results on faces in $\mathbb{M}_{1}^{+}(K)$ which may also be of inderendent interest.

Definition: If $C$ is a convex set and $F$ is a subset of $C$, then the set

$$
F^{\prime}=U\{G: G \text { a face in } C \text { and } G \cap F=\varnothing\}
$$

is called the complementary set of $\underline{F}$.

Lemma 2.1: Let $F \neq \varnothing$ be a face in $\mathbb{M}_{1}^{+}(K)$, and let $u \in \mathbb{M}_{1}^{+}(K)$. Then $u \in F^{\prime}$ if and only if $\mu \perp \nu$ for all $\nu \in F$.

Proof: 1) If $u+\nu$ for all $\nu \in F$, then obviously face $(\mu) \cap F=\varnothing$, so $\mu \in F^{\prime}$.

2) Suppose next that there is a $\nu \in F$ such that $u \not \perp \nu$. Let $\mu=\mu_{a}+\mu_{S}$ be the Lebesgue-decomposition of $\mu$ w.r.t. $\nu$. Then $\mu_{a} \neq 0$. Let $f=\frac{d \mu_{a}}{d \nu} \in I^{1}(\nu)$, and define

$$
B=\left\{x \in K: f(x) \leq\|f\|_{L^{1}(\nu)}\right\} \text {. }
$$

Clearly $B$ is measurable and $\int_{B} f d \nu>0$.

Define $g=f \cdot x_{B}$ and $h={ }^{B} f \cdot x_{K} B$. Then $g \in I^{\infty}(\nu)$ and $\mu=g \cdot \nu+h \cdot \nu+\mu_{S}$. Hence we have $(\nu(g))^{-1} \cdot g \cdot \nu \in F \cap$ face $(\mu)$. Hence $\mu \not F^{\prime}$, and the proof is complete.

Corollary 2.2: If $\mathrm{F} \neq \varnothing$ is a face in $\mathbb{M}_{1}^{+}(\mathrm{K})$, then $\mathrm{F}$. is a face.

Definition: Let $C$ be a convex set and $F$ a face of $C$. 
We shall say that $F$ is a split face of $C$ if $F^{\prime}$ is a face of $C$ and every $\mathrm{x} \in \mathrm{C}\left(\mathrm{F} \cup \mathrm{F}^{\mathrm{i}}\right)$ has a unique decomposition

$$
\mathrm{x}=\lambda \mathrm{y}+(1-\lambda) \mathrm{z} ; \mathrm{y} \in \mathrm{F}, \mathrm{z} \in \mathrm{F}^{\prime} \text { and } \lambda \in\langle 0,1\rangle \text {. }
$$

Note that if $F$ is a split face of $C$, then necessarily $C=c o\left(F \cup F^{\prime}\right)$. For convex sets of the particular form $M_{1}^{+}(K)$, this is a sufficient condition that a face $F$ be split.

Corollary 2.3: Let $F$ be a face in $\mathbb{M}_{1}^{+}(K)$. Then $F$ is a split face if and only if $\operatorname{co}\left(\mathrm{F} \cup \mathrm{F}^{\prime}\right)=\mathrm{M}_{1}^{+}(\mathrm{K})$.

Proof: We only need to show that if $\mu \in \operatorname{co}\left(F \cup F^{\prime}\right) 、\left(F \cup F^{\prime}\right)$, then $\mu$ has a unique decomposition after $F$ and $F^{\prime}$. Suppose $\mu_{1}, \nu_{1} \in F, \mu_{2}, \nu_{2} \in F^{\prime}$ and $\alpha, \lambda \in<0,1>$ are such that

$$
\mu=\lambda \mu_{1}+(1-\lambda) \mu_{2}=\alpha \nu_{1}+(1-\alpha) \nu_{2} \text {. }
$$

Since $\left(\lambda \mu_{1}-\alpha \nu_{1}\right) \perp\left((1-\lambda) \mu_{2}-(1-\alpha) \nu_{2}\right)$, we have

$$
0=\left\|\lambda \mu_{1}-\alpha \nu_{1}\right\|+\left\|(1-\lambda) \mu_{2}-(1-\alpha) \nu_{2}\right\|
$$

Hence $\lambda=\alpha, \mu_{1}=\nu_{1}$ and $\mu_{2}=\nu_{2}$, and the proof is complete.

Theorem 2.4: Let $F \neq \varnothing$ be a face in $\mathbb{M}_{1}^{+}(K)$. Then $F$ is a split face if and only if $F$ is norm closed.

Proof: 1) Suppose $F$ is a split face. Define a function $f: M_{1}^{+}(K) \rightarrow[0,1]$ by setting $f(x)=\lambda$, where $\lambda$ is given by

$$
\mathrm{x}=\lambda \mathrm{y}+(1-\lambda) \mathrm{z} ; \mathrm{y} \in \mathrm{F}, \mathrm{z} \in \mathrm{F}^{\prime} \text { and } \lambda \in[0,1] \text {. }
$$

The function $f$ is affine, and it is elementary and well known that $f$ has a unique extension to a linear functional $f$ ' on $M(K)$ - This $f^{\prime}$ is bounded on the unit ball in $M(K)$, and 
hence $f^{\prime}$ is norm continuous. Thus, $F=f^{-1}(1) \cap M_{1}^{+}(K)$ is norm closed.

2) Suppose $F$ is norm closed. From the proof of theorem 2.7. in [9], it follows that if $\mu \in \mathbb{M}_{1}^{+}(K)$, then there exists $\nu \in F$ such that if $\mu=\mu_{a}+\mu_{s}$ is the Lebesgue-decomposition of $\mu$ w.r.t. $\nu$, then $\mu_{S} \perp \sigma$ for all $\sigma \in F$. Hence $\mu_{S}=0$ or $\left\|\mu_{S}\right\|^{-1} \cdot \mu_{S} \in F^{\prime}$ by Iemma 2.1. Now it is clear that $\mathbb{M}_{1}^{+}(K)=$ $c o\left(F \cup F^{r}\right)$, and the proof is complete.

Remarks: Corollary 2.2., Corollary 2.3. and Theorem 2.4. are contained in the papers [2] of Alfsen and [6] of Asinow-Ellis. The proofs seems new apart from the first half of the proof of Theorem 2.4. The second half of theorem 2.4. also follows from Prop.2.12. in [5].

Theorem 2.5: Let $\mathrm{F} \neq \varnothing$ be a face in $\mathrm{M}_{1}^{+}(\mathrm{K})$. Then $\overline{\mathrm{F}}^{\text {norm }}$ is a face in $\mathbb{M}_{1}^{+}(K)$ and $\mathrm{F}^{\prime}=\left(\overline{\mathrm{F}}^{\text {norm }}\right)^{\prime}$.

Proof: Let $\mu_{1}, \mu_{2} \in \mathbb{M}_{1}^{+}(K)$ and $\lambda \in\langle 0,1\rangle$ be such that $\mu=\lambda \mu_{1}+(1-\lambda) \mu_{2} \in \bar{F}^{\text {norm }}$. Let $\left\{\mu_{n}\right\} \subseteq F$ be such that $\left\|\mu-\mu_{n}\right\| \rightarrow 0$, as $n \rightarrow \infty$. Let $\mu_{n}=\mu_{n a}+\mu_{n s}$ be the Lebesgue-decomposition of $\mu_{n}$ w.r.t. $\mu$. Then $\left\|\mu_{n s}\right\| \rightarrow 0$, and $\left\|\mu-\mu_{n a}\right\| \rightarrow 0$, as $n \rightarrow \infty$. By Proposition 1.2. there is a $f \in I^{\infty}(\mu)$ such that $f \cdot \mu=\mu_{1}$. Define measures $\nu_{n}$ by

$$
\nu_{n}=\left(u_{n a}(f)\right)^{-1} \cdot\left\|\mu_{n a}\right\| \cdot f \cdot \mu_{n a}+\mu_{n s}
$$

Then $\nu_{n} \in \mathrm{F}$ and we have

$$
\left|1-\mu_{n a}(f)\right|=\|\| f \cdot \mu\|-\| f \cdot \mu_{n a}\|\leq\| f\left\|_{b}\right\| \mu-\mu_{n a} \| \rightarrow 0 \text {, as } n \rightarrow \infty \text {. }
$$

Hence

$$
\begin{aligned}
& \left\|u_{1}-\nu_{n}\right\|=\left\|f \cdot \mu-\left(\mu_{n a}(f)\right)^{-1} \cdot\right\| \mu_{n a}\left\|\cdot f \cdot \mu_{n a}-\mu_{n s}\right\| \\
\leq & \left\|\mu_{n s}\right\|+\|f\|_{\infty}\left\|\mu-\mu_{n a}\right\|+\mid 1-\mu_{n a}(f)^{-1}\left\|\mu_{n a}\right\| \cdot\left\|f \mu_{n a}\right\| \rightarrow 0 \text {, as } n \rightarrow \infty
\end{aligned}
$$


Thus $\mu_{1} \in \overline{\mathrm{F}}^{\text {norm }}$, and $\overline{\mathrm{F}}^{\text {norm }}$ is a face.

Obtiously we have $\left(\bar{F}^{\text {norm }}\right), \subseteq F^{\prime}$.

Let $\nu \in F^{\prime}$, and let $\mu \in \bar{F}^{\text {norm }}$. Let $\left\{\mu_{n}\right\} \subseteq F$ be such that $\left\|\mu-\mu_{n}\right\| \rightarrow 0$ as $n \rightarrow \infty$. Then

$$
\|\nu-\mu\| \geq\left\|\nu-u_{n}\right\|-\left\|\mu-\mu_{n}\right\|=2-\left\|\mu-\mu_{n}\right\| \rightarrow 2 \text {, as } n \rightarrow \infty \text {. }
$$

Hence $\|\nu-u\|=2$, so $\nu \perp \mu$. From Iemma 2.1. it now follows that $F^{\prime} \subseteq\left(\bar{F}^{\text {norm }}\right) \cdot$, and the proof is complete.

It is well known that $K$ is affinely homoemorphic to a $\mathrm{w}^{*}$ compact convex subset of $A(K) *[3]$. By the norm topology on $K$ we will mean the relative topology of $K$ as a subset of the Banach space $A(K) *$.

Lemma 2.6: Let $K$ be a compact simplex. For each $z \in \mathbb{K}$, let $\mu_{z}$ be the unique measure in $Q_{z}$. Then for every $x$ and every $y \in K$, we have $\|x-y\|=\left\|\mu_{x}-\mu_{y}\right\|$.

Proof: We have

$$
\begin{aligned}
& \|x-y\|=\sup _{\substack{\|f\| \leq 1 \\
f \in A(K)}}|f(x)-f(y)|=\sup _{\substack{\|f\| \leq 1 \\
f \in A(K)}}\left|\mu_{x}(f)-\mu_{y}(f)\right| \\
& \leq \sup _{\substack{\|f\| \leq 1 \\
f \in C(K)}}\left|\mu_{X}(f)-\mu_{y}(f)\right|=\left\|\mu_{X}-\mu_{y}\right\|
\end{aligned}
$$

Let $\varepsilon>0$ and let $f \in C(K)$ with $\|f\| \leq 1$ be such that

$$
\left\|\mu_{x}-\mu_{y}\right\| \leq\left|\mu_{x}(f)-\mu_{y}(f)\right|+\epsilon
$$

By Iemma 9.2. and Proposition 9.3. in [15] we can find convex functions $g_{1}, g_{2} \in C(K)$ and concave functions $f_{1}, f_{2} \in C(K)$ such that $g_{1}, g_{2} \leq f \leq f_{1}, f_{2}$ and

$$
\left|\mu_{x}\left(g_{1}\right)-\mu_{x}\left(f_{1}\right)\right|<\epsilon
$$


and

$$
\left|\mu_{y}\left(g_{2}\right)-\mu_{y}\left(f_{2}\right)\right|<\epsilon
$$

By a theorem of Edwards [8] we can find a $h \in A(K)$ such that

$$
\operatorname{maks}\left(-1, g_{1}, g_{2}\right) \leq h \leq \min \left(1, f_{1}, f_{2}\right) \text {. }
$$

Thus

$$
\left|\mu_{X}(f)-h(x)\right|=\left|\mu_{X}(f)-\mu_{X}(h)\right|<2 \epsilon
$$

and

$$
\left|\mu_{y}(f)-h(y)\right|=\left|\mu_{y}(f)-\mu_{y}(h)\right|<2 \epsilon \text {. }
$$

Hence

$$
\left\|\mu_{x}-u_{y}\right\| \leq|h(x)-h(y)|+5 \epsilon \leq\|x-y\|+5 \epsilon
$$

Since $€$ was arbitrary,

$$
\|x-y\|=\left\|\mu_{x}-\mu_{y}\right\|
$$

and the proof is complete.

Corollary 2.7: Let $K$ be a compact simplex, and let $F \neq \varnothing$ be a face of $K$. Then $\bar{F}^{\text {norm }}$ is a face of $K$, and $F^{\prime}=\left(\bar{F}^{\text {norm }}\right)^{\prime}$.

Proof: It is well known that $Q_{1}$ is a norm closed face of $M_{1}^{+}(K)$. When $K$ is a compact simplex, $r$ is a bijection between $Q_{1}$ and $K$. From Lemma 2.6. it follows that a subset $S$ of $Q_{1}$ is norm closed if and only if $r(S)$ is norm closed. Now the Corollary follows from Theorem 2.5., and the proof is complete.

Theorem 2.8: (Alfsen, Asimow, Ellis) Let $K$ be a compact simplex and let $F \neq \varnothing$ be a face of $K$. The following statements are equivalent:

(i) $\quad F$ is a split face.

(ii) $K=\operatorname{co}\left(F \cup F^{\prime}\right)$ 
(iii) $F$ is norm closed.

(iv) $r^{-1}(F) \cap Q_{1}$ is norm closed.

(v) $\quad r^{-1}(F) \cap Q_{1}$ is a split face.

Proof: $(i) \Leftrightarrow(v)$ is obvious since the restriction of $r$ to $Q_{1}$ is a bijection.

(v) $\Longleftrightarrow$ (iv) follows from theorem 2.4.

(iv) $\Longleftrightarrow$ (iii) follows from Lemma 2.6.

(i) $\Rightarrow$ (ii) is trivial.

(ii) $\Longrightarrow(v)$ follows from Corollary 2.3.

The proof is complete.

Remarks: 1. (i) $\Longleftrightarrow$ (ii) in Theorem 2.8. is first proved by Alfsen in [2]. (ii) $\Longleftrightarrow$ (iii) is first proved by AsimowEllis in [6].

2. It is well known that $\mathbb{M}(K)$ is an order complete vector lattice. In order complete vector lattices, the notion of a band has a meaning. The notion of a band was introduced by Riesz (See [16] or [14]). It can be shown that the map $F \rightarrow$ linspan $F$ is a bijection (with inverse $\mathbb{M} \rightarrow \mathbb{M} \cap \mathbb{M}_{1}^{+}(K)$ ) between the split faces of $M_{1}^{+}(K)$ and the bands in $M(K)$.

From Corollary 1.4. and Theorem 2.4. we get that if $\mu \in \mathbb{M}_{1}^{+}(K)$, then the smallest band containing $\mu$ is given by Iin $\operatorname{span}\left(\overline{\text { face }(\mu)^{\text {norm }}}\right)=\{\nu \in \mathbb{M}(K): \nu \ll \mu\}$.

3. More results on split faces can be found in [3], [4], $[6],[10],[12],[13],[17]$.

Example: Let $\mathrm{K}=[0,1]$ and define $F=\left\{\mu \in \mathbb{M}_{1}^{+}(K): \mu(\{x\})=0\right.$, all $\left.x \in[0,1]\right\}$ 
(i) $F$ is a split face. Hence $F$ is norm-closed.

(ii) $\partial_{e} F=\varnothing$.

(iii) $F$ is a $G_{\delta}$ subset of $M_{1}^{+}(K)$.

(iv) It does not exist any Borel set $B \subseteq[0,1]$ such that $\mu(B)=1$ for all $\mu \in F$, and $\mu(B)=0$ for all $\mu \in F^{\prime}$.

Proof: (i) is clear.

(ii) $\quad \partial_{e} F \subseteq e^{\mathbb{M}_{1}^{+}(K)}=\left\{\epsilon_{x}: x \in[0,1]\right\}$. Hence $\partial_{e} F=\varnothing$.

(iii) See Proposition I.2.8. and Example I.2.10. in [3].

(iv) is clear since $\left\{\epsilon_{\mathrm{x}}: \mathrm{x} \in[0,1]\right\} \subseteq \mathrm{F}^{\prime}$. 


\section{Characterization of split faces by strongly disjointness}

Definition: Iet $B$ and $D$ be two convex subsets of the vector space $E$, and let $G=c o(B \cup D)$. We shall say that $B$ and $D$ are affinely independent if every $x \in G$ can be expressed by a unique convex combination

$$
x=\lambda y+(1-\lambda) z, y \in B, z \in D, \lambda \in[0,1] .
$$

Definition: Let $C$ be a convex subset of the vector space $E$, and let $F, G$ be two faces of $C$. We shall say that $F$ and $G$ are strongly disjoint, and we shall write $F b G$, if $F$ and $G$ are affinely independent and if $c o(F \cup G)$ is a face of $C$.

We shall say that two points $\mathrm{x}, \mathrm{y}$ of $\mathrm{C}$ are strongly disjoint, and we shall write $\mathrm{x} \delta \mathrm{y}$, if face $(\mathrm{x}) \mathrm{d}$ face $(\mathrm{y})$.

In [12] the following was proved:

Proposition 3.1: Let $C$ be a convex subset of $E$ and let $F \neq \varnothing$ be a face of $C$. The following statements are equivalent: (i) $F$ is a split face.

(ii) $\quad C=c o\left(F \cup F^{\prime}\right)$ and if $z \in F^{\prime}$, then Foface $(z)$. (iii) $C=c o\left(F \cup F^{\prime}\right)$ and for every $x \in F$ and every $y \in F^{\prime}$, $\mathrm{x} 6 \mathrm{y}$.

Proposition 3.2: Let $C$ be a linearly compact convex subset of $E$ and let $F \neq \varnothing$ be a face of $C$. The following statements are equivalent:

(i) $F$ is a split face.

(ii) $\quad C=c o\left(F^{\prime} \cup F^{\prime}\right)$ and if $z \in F^{\prime}$, then $c o(F \cup$ face $(z))$ is a face of $C$. 
(iii) $C=\operatorname{co}\left(F \cup F^{\prime}\right)$ and for every $\mathrm{x} \in \mathrm{F}$ and every $\mathrm{y} \in \mathrm{F}^{\prime}$, co(face $(x)$ Uface $(y))$ is a face of $C$.

Proof: Suppose $G$ is a face of $F$ such that if $z \in F^{\prime}$, then co(GUface $(z))$ is a face of $C$. Then it is enough to show that $G$ and face $(z)$ are affinely independent.

Let $x \in \operatorname{co}(G \|$ face $(z)) \backslash(G \cup$ face $(z))$ and suppose there exist $y_{i} \in G, u_{i} \in \operatorname{face}(z)$ and $\lambda_{i} \in<0,1>$ such that

$$
x=\lambda_{i} y_{i}+\left(1-\lambda_{i}\right) u_{i} \quad, i=1,2 \text {. }
$$

We may assume that $\left(1-\lambda_{2}\right) \geq\left(1-\lambda_{1}\right)$. Since co(GUface $\left.\left(u_{1}\right)\right)$ is a face, we have $y_{2}, u_{2} \in \operatorname{co}\left(G U\right.$ face $\left.\left(u_{1}\right)\right)$. Hence $u_{2} \in$ face(u). Suppose $u_{2} \neq u_{1}$. Then

$$
u_{1}=\beta u_{2}+(1-\beta) z^{\prime}
$$

for some $z^{\prime} \in C$ and some $\beta \in\langle 0,1\rangle$.

Let $I$ be the line through $u_{1}$ and $u_{2}$. Then $I \cap C$ is a closed interval, and face $\left(u_{1}\right)$ intersects the interior of $I \cap C$. Hence $I \cap C \subseteq$ face $\left(u_{1}\right)$. Without loss of generality, we may assume $z^{\prime}$ is an endpoint of the interval $I \cap C$. Then we have $u_{1}, u_{2} \notin$ face $\left(z^{1}\right)$. Now

$$
\begin{aligned}
& {\left[1-\beta\left(1-\lambda_{1}\right)\right]^{-1}\left(\lambda_{2} \mathrm{y}_{2}+\left[\left(1-\lambda_{2}\right)-\beta\left(1-\lambda_{1}\right)\right] u_{2}\right) } \\
= & {\left[1-\beta\left(1-\lambda_{1}\right)\right]^{-1}\left(\lambda_{1} \mathrm{y}_{1}+\left(1-\lambda_{1}\right)(1-\beta) z^{p}\right) \in \operatorname{co}\left(G \text { Uface }\left(z^{\prime}\right)\right) . }
\end{aligned}
$$

Hence $u_{2} \in$ face $\left(z^{9}\right)$. Thus we have obtained a contradiction. Hence $u_{2}=u_{1}$, and we have:

$$
\lambda_{1} \mathrm{y}_{1}=\lambda_{2} \mathrm{y}_{2}+\left[\left(1-\lambda_{2}\right)-\left(1-\lambda_{1}\right)\right] u_{2}
$$

Hence $\lambda_{1}=\lambda_{2}$ and $\mathrm{y}_{1}=\mathrm{y}_{2}$, and the proof is complete. 
Definition: A measure $\mu \in \mathbb{M}_{1}^{+}(K)$ is said to be central if for any Borel set $B \subseteq K$ such that $\lambda=\mu(B) \in\langle 0,1\rangle$, the barycenters of the probability measures $\left.\lambda^{-1} \mu\right|_{B}$ and $\left.(1-\lambda)^{-1} \mu\right|_{K} \cdot B$ are strongly disjoint.

Remark: The concept of a central measure was introduced by Sakai [18], [19] for $C^{*}$-algebras, and extended by Wils [20], [21], [22] to general compact convex sets. See also Alfsen [3].

Theorem 3.3: Let $F \neq \varnothing$ be a face in the compact convex set $K$. The following statements are equivalent:

(i) $F$ is a split face

(ii) For each $x \in K$, there exist $y \in F, z \in F^{\prime}$ and $\lambda \in[0,1]$ such that $\mathrm{x}=\lambda \mathrm{y}+(1-\lambda) \mathrm{z}$ and $\mathrm{y} d \mathrm{z}$.

(iii) For each $\mathrm{x} \in \mathrm{K}$, there exists a central measure $\nu \in \mathbb{M}_{\mathrm{X}}$ such that $\nu=\lambda \nu_{1}+(1-\lambda) \nu_{2}$ where $\lambda \in[0,1], r\left(\nu_{1}\right) \in \mathbb{F}$ and $r\left(\nu_{2}\right) \in F^{\prime}$.

(iv) If $x \in K$ and $\mu \in Q_{X}$, then $\mu$ has a decomposition $\mu=\lambda \mu_{1}+(1-\lambda) \mu_{2}$ such that $\lambda \in[0,1], r\left(\mu_{1}\right) \in F$ and $r\left(\mu_{2}\right) \in F^{\prime}$, and $r\left(\mu_{1}\right)$ is fixed as $\mu$ is varying through $Q_{x} \cdot$

Proof: (i) $\Rightarrow$ (ii) See Proposition 3.1.

(ii) $\Rightarrow$ (iii) It is seen that if $\mathrm{x}=\lambda \mathrm{y}+(1-\lambda) \mathrm{z}$ with $\mathrm{y} \in \mathrm{F}$, $z \in F^{\prime}$ and $y \dot{z} z$, then $\nu=\lambda \epsilon_{y}+(1-\lambda) e_{z}$ fulfills the requirements to the measure $v$ in (iii). (iii) $\Rightarrow$ (iv) Let $x \in K$ and let $\mu \in Q_{X}$. Let $\nu \in \mathbb{M}_{X}$ satisfy the requirements of (iii). Since $\nu$ is central we have by Corollary 4.7, that $\nu<\mu$. (See also [22].) By Corollary I.3.3. 
in [3] there exists $\mu_{1}, \mu_{2} \in \mathbb{M}_{1}^{+}(K)$ such that $\nu_{1}<\mu_{1}, \nu_{2}<\mu_{2}$ and

$$
\mu=\lambda \mu_{1}+(1-\lambda) \mu_{2}
$$

Since $\nu_{1}$ and $\nu_{2}$ are in disjoint faces of $M_{1}^{+}(K)$, we have by Lemma 2.1. that $\nu_{1} \perp v_{2}$. Thus we can find a Borel set $B \subseteq K$ such that $\nu_{1}(B)=1$ and $\nu_{2}(K, B)=1$. Hence, since $\nu$ is central, $r\left(\nu_{1}\right) b r\left(\nu_{2}\right)$. Now the uniqueness of $r\left(u_{1}\right)$ easily follows.

$(i v) \Rightarrow(i)$ It is clear that $K=c o\left(F \cup F^{\prime}\right)$, and that every $\mathrm{X} \in \mathrm{K}$ has a unique decomposition after $F$ and $F^{\prime}$. Thus we only need to show that $F^{\prime}$ is convex.

Suppose $\mathrm{y}_{1}, \mathrm{y}_{2} \in \mathrm{F}^{\prime}, \lambda \in<0,1>$ and

$$
\mathrm{y}=\lambda \mathrm{y}_{1}+(1-\lambda) \mathrm{y}_{2} \notin \mathrm{F}^{\prime}
$$

Let $\nu_{1} \in \mathrm{Qy} \mathrm{y}_{1}$ and $\nu_{2} \in \mathrm{Qy}_{2}$. Then $\nu=\lambda \nu_{1}+(1-\lambda) \nu_{2} \in \mathrm{Qy}$, and by (iv)

$$
\nu=\alpha \mu_{1}+(1-\alpha) \mu_{2}
$$

where $r\left(\mu_{1}\right) \in F, r\left(\mu_{2}\right) \in F^{\prime}$ and $\alpha \in<0,1>$. By Proposition II.3.3. in [3], there exist $\partial_{i j} \geq 0$ and $\pi_{i j} \in \mathbb{M}_{1}^{+}(K)$ such that

$$
\lambda \nu_{1}=\partial_{11} \pi_{11}+\partial_{12} \pi_{12},(1-\lambda) \nu_{2}=\partial_{21} \pi_{21}+\partial_{22} \pi_{22}
$$

and

$$
\alpha \mu_{1}=\partial_{11} \pi_{11}+\partial_{21} \pi_{21},(1-\alpha) \mu_{2}=\partial_{12} \pi_{12}+\partial_{22} \pi_{22} .
$$

Since $\alpha>0$, we have $\partial_{11}+\partial_{21}>0$. If $\partial_{11} \neq 0$, we have $r\left(\pi_{11}\right) \in$ face $\left(y_{1}\right)$, and if $\partial_{21} \neq 0$, we have $r\left(\pi_{21}\right) \in$ face $\left(y_{2}\right)$. Thus face $\left(r\left(\mu_{1}\right)\right) \cap F^{\prime} \neq \varnothing$. This is a contradiction, so $F^{9}$ is convex, and the proof is complete.

Remark: Theorem 3.3. extends Theorem 2.17. in [10] and Theorem II.6.12. in [3]. 
The next result extends Theorem II.6.18. in [3].

Corollary 3.4: Let $F \subseteq K$ be a face. The following statements are equivalent:

$F$ is a split face

(ii) $K=c o\left(F \cup F^{\circ}\right)$ and for each $f \in A(K)$, there exist a bounded affine function $g$ on $K$ such that $g=f$ on $F$ and $g=0$ on $F^{\prime}$.

Proof: $(i) \Rightarrow$ (ii) $K=c o\left(F \cup F^{\prime}\right)$ follows from the definition of a split face.

Suppose $f \in A(K)$, and let $x \in K$. Let $\mu \in Q_{X}$. By (iv) in Theorem 3.3. we have

$$
\mu=\lambda \mu_{1}+(1-\lambda) \mu_{2}
$$

where $r\left(\mu_{1}\right) \in F$ and $r\left(\mu_{2}\right) \in F^{\prime}$. Define $g(x)$ by

$$
g(x)=\lambda f\left(r\left(\mu_{1}\right)\right) \text {. }
$$

By (iv) in Theorem 3.3. $g$ is well-defined. Also $g$ is bounded, affine and aatisfies $g=f$ on $F$ and $g=0$ on $F^{\prime}$. (ii) $=>$ (i) Let $g$ be an affine function on $K$ such that $g=1$ on $F$ and $g=0$ on $F^{9}$. Then $g \geq 0$ on $K$ and $g^{-1}(0)$ is a face disjoint from $F^{\prime}$ containing $F^{\prime}$, so $F^{\prime}=g^{-1}(0)$. Hence $F^{\prime}$ is face.

Let $x \in K\left(F \cup F^{\prime}\right)$ and suppose $x=\lambda_{i} y_{i}+\left(1-\lambda_{i}\right) z_{i}, y_{i} \in F, z_{i} \in F^{\prime}, \lambda_{i} \in<0,1>, \quad i=1,2$. If $y_{1} \neq y_{2}$, then there exist an $f \in A(K)$ such that $f\left(y_{1}\right)=1$ and $f\left(y_{2}\right)=0$. Let $g$ be bounded and affine such that $g=f$ on $F$ and $g=0$ on $F^{\prime}$. Then $g(x)=\lambda_{1} \cdot 1=\lambda_{2} \cdot 0$. This is a contradiction, so $y_{1}=y_{2}$. Now it is easy to see that $\lambda_{1}=\lambda_{2}$ and $z_{1}=z_{2}$, and the proof is complete. 
4. On properties of central measures

We begin with some lemmas.

Lemma 4.1: Let $\mathrm{x}_{1}, \mathrm{x}_{2} \in \mathrm{K}, \lambda \in\langle 0,1\rangle$ and $\mathrm{x}=\lambda \mathrm{x}_{1}+(1-\lambda) \mathrm{x}_{2}$. If the decomposition of $x$ after face $\left(x_{1}\right)$ and face $\left(x_{2}\right)$ is unique, then face $\left(x_{1}\right)$ and face $\left(x_{2}\right)$ are affinely independent.

Proof: Define $G=c o\left(\right.$ face $\left(x_{1}\right) \cup$ face $\left.\left(x_{2}\right)\right)$, and let $z \in G$. We shall show that $z$ has a unique decomposition after face $\left(x_{1}\right)$ and face $\left(x_{2}\right)$.

Since $z \in G$, we can find $\partial \in[0,1], y_{1} \in$ face $\left(x_{1}\right)$ and $y_{2} \in$ face $\left(x_{2}\right)$ such that

$$
\mathrm{z}=\partial \mathrm{y}_{1}+(1-\partial) \mathrm{y}_{2} \cdot
$$

Let $\left.\alpha_{i}<0,1\right]$ and $u_{i} \in$ face $\left(x_{i}\right)$ be such that

$$
x_{i}=x_{i} y_{i}+\left(1-\alpha_{i}\right) u_{i} \quad, i=1,2 \text {. }
$$

Then

$$
\mathrm{x}=\lambda \alpha_{1} \mathrm{y}_{1}+(1-\lambda) \alpha_{2} \mathrm{y}_{2}+\lambda\left(1-\alpha_{1}\right) \mathrm{u}_{1}+(1-\lambda)\left(1-\alpha_{2}\right) \mathrm{u}_{2}
$$

Let $1>\epsilon>0$ be such that $\lambda \alpha_{1} \geq \partial \epsilon$ and $(1-\lambda) \alpha_{2} \geq(1-\partial) \varepsilon$. Then we have

$$
x=\epsilon z+(1-\epsilon) y
$$

where $y \in G \cap$ face $(x)$ and $(1-\epsilon) \mathrm{y}=\left(\lambda \alpha_{1}-\partial \epsilon\right) \mathrm{y}_{1}+\left((1-\lambda) \alpha_{2}-(1-\partial) \epsilon\right) \mathrm{y}_{2}+\lambda\left(1-\alpha_{1}\right) u_{1}+(1-\lambda)\left(1-\alpha_{2}\right) u_{2}$.

Suppose

$$
z=\gamma y_{3}+(1-\gamma) y_{4}
$$

where $\gamma \in[0,1], y_{3} \in$ face $\left(x_{1}\right)$ and $y_{4} \in$ face $\left(x_{2}\right)$. We shall show that $y=\partial, y_{3}=y_{1}$ and $y_{4}=y_{2}$. 
Let $\beta \in[0,1], v_{1} \in$ face $\left(x_{1}\right)$ and $v_{2} \in$ face $\left(x_{2}\right)$ be such that

$$
\mathrm{y}=\beta \mathrm{v}_{1}+(1-\beta) \mathrm{v}_{2}
$$

Then we have

$$
\begin{aligned}
\mathrm{x} & =\epsilon \partial \mathrm{y}_{1}+(1-\epsilon) \beta \mathrm{v}_{1}+\epsilon(1-\partial) \mathrm{y}_{2}+(1-\epsilon)(1-\beta) \mathrm{v}_{2} \\
& =\epsilon \gamma \mathrm{y}_{3}+(1-\epsilon) \beta \mathrm{v}_{1}+\epsilon(1-\gamma) \mathrm{y}_{4}+(1-\epsilon)(1-\beta) \mathrm{v}_{2} .
\end{aligned}
$$

Since $x$ has a unique decomposition after face $\left(x_{1}\right)$ and face $\left(x_{2}\right)$, we now get

$$
\epsilon \partial+(1-\epsilon) \beta=\epsilon \gamma+(1-\epsilon) \beta
$$

and

$$
\epsilon \partial \mathrm{y}_{1}+(1-\epsilon) \beta \mathrm{v}_{1}=\epsilon \gamma u_{1}+(1-\epsilon) \beta \mathrm{v}_{1}
$$

Hence

$$
\partial=\gamma \text { and } y_{3}=y_{1}
$$

This shows that $z$ has a unique decomposition after face $\left(\mathrm{x}_{1}\right)$ and face $\left(x_{2}\right)$, and the proof is complete.

Iemma 4.2: Let $\mathrm{x}_{1}, \mathrm{x}_{2} \in \mathrm{K}, \lambda \in\langle 0,1\rangle$ and $\mathrm{x}=\lambda \mathrm{x}_{1}+(1-\lambda) \mathrm{x}_{2}$. If $\lambda \epsilon_{\mathrm{x}_{1}}+(1-\lambda) \epsilon_{\mathrm{x}_{2}}<\nu$ for all $\nu \in \mathrm{Q}_{\mathrm{X}}$, then face $(\mathrm{x})=$ co face $\left(x_{1}\right) \cup$ face $\left.\left(x_{2}\right)\right)$.

Proof: We have to show that face $(x) \subseteq \operatorname{co}\left(\right.$ face $\left(x_{1}\right) \cup$ face $\left.\left(x_{2}\right)\right)$. Let $z_{1} \in$ face $(x)$ and let $\left.\alpha \in<0,1\right]$ and $z_{2} \in$ face $(x)$ be such that

$$
x=\alpha z_{1}+(1-\alpha) z_{2}
$$

Define

$$
\nu=\alpha \epsilon_{z_{1}}+(1-\alpha) \epsilon_{z_{2}}
$$

and let $\pi \in Q_{\mathrm{X}}$ be such that $\nu<\pi$. Since $\lambda \epsilon_{\mathrm{x}_{1}}+(1-\lambda) \epsilon_{\mathrm{x}_{2}}<\pi$, we can find $\tau_{i} \in \mathbb{M}_{x_{i}}$ and $\eta_{i} \in \mathbb{N}_{z_{i}}([3$; Corollary 1.3 .3$])$, 
$i=1,2$, such that

$$
\begin{aligned}
\pi & =\lambda \tau_{1}+(1-\lambda) \tau_{2} \\
& =\alpha \eta_{1}+(1-\alpha) \eta_{2}
\end{aligned}
$$

By Proposition II.3.3. in [3] there exist $\partial_{i} \geq 0$ and $\pi_{i} \in \mathbb{M}_{1}^{+}(K)$, $i=1, \ldots, 4$, such that

$$
\lambda \tau_{1}=\partial_{1} \pi_{1}+\partial_{2} \pi_{2},(1-\lambda) \tau_{2}=\partial_{3} \pi_{3}+\partial_{4} \pi_{4}
$$

and

$$
\alpha \eta_{1}=\partial_{1} \pi_{1}+\partial_{3} \pi_{3},(1-\alpha) \eta_{2}=\partial_{2} \pi_{2}+\partial_{4} \pi_{4} .
$$

Hence we have

$$
z_{1}=r\left(\eta_{1}\right)=\alpha^{-1} \partial_{1} r\left(\pi_{1}\right)+\alpha^{-1} \partial_{3} r\left(\pi_{3}\right)
$$

So $z_{1} \in \operatorname{co}\left(\right.$ face $\left(x_{1}\right)$ uface $\left.\left(x_{2}\right)\right)$, and the proof is complete.

The proof of the next two lemmas can be found in [3; Chap.II, $\S 8]$.

Iemma 4.3: Let $\mu \in \mathbb{M}_{1}^{+}(K)$ be a central measure, and let $\left\{B_{1}, \ldots, B_{n}\right\}$ be a Borel partition of $I$ such that $\lambda_{i}=\mu\left(B_{i}\right) \epsilon$ $<0,1\rangle$ for $i=1, \ldots, n$. If $x_{i}=r\left(\lambda_{i}^{-1} \mu_{B_{i}}\right)$ for $i=1, \ldots, n$, then the measure

$$
\nu=\sum_{i=1}^{n} \lambda_{i} \epsilon_{x_{i}}
$$

is central.

Iemma 4.4: Let $\mu \in \mathbb{M}_{x}$ be a central measure. Then there exist a net $\left\{\mu_{\alpha}\right\} \subseteq \mathbb{M}_{x}$ of discrete central measures such that $\mu_{\alpha}<\mu$ for all $\alpha$ and $\mu_{\alpha} \rightarrow \mu$ w*

Lemma 4.5: Let $\mu=\sum_{i=1}^{n} \lambda_{i} \epsilon_{x_{i}} \in \mathbb{M}_{x}$ be a central measure. 
$\left(\lambda_{i}>0\right.$ all $\left.i\right)$. If $x=\sum_{j=1}^{m} \alpha_{j} y_{j}\left(\alpha_{j}>0\right.$ all $\left.j\right)$, then there exist $\gamma_{i j} \geq 0, z_{i j} \in K(i=1, \ldots, n ; j=1, \ldots, m)$ such that

$$
\lambda_{i} x_{i}=\sum_{j=1}^{m} \gamma_{i j} z_{i j} \quad, \alpha_{j} y_{j}=\sum_{i=1}^{n} \gamma_{i j} z_{i j} \text {. }
$$

If we define $z_{i j}=x$ when $\gamma_{i j}=0$, then the above decomposition is unique.

Proof: It is clear from the definition of a central measure that face $\left(x_{i}\right)$ is a split face in face $(x)$ for each $i$. Thus $\operatorname{co}\left(\bigcup_{i=1}^{n}\right.$ face $\left.\left(x_{i}\right)\right)$ is a face (See Proposition 3.2. or [3; Corollary II.6.8]) containing $x$, and hence

$$
\text { face }(x)=\operatorname{co}\left(\bigcup_{i=1}^{n} \text { face }\left(x_{i}\right)\right) \text {. }
$$

For each $y_{j}$, we can find $\gamma_{i j} \geq 0$ and $z_{i j} \in$ face $\left(x_{i}\right)$ such that

$$
\alpha_{j} \mathrm{y}_{j}=\sum_{i=1}^{n} \gamma_{i j} z_{i j}
$$

and such that $z_{i j}=x$ if $\gamma_{i j}=0$.

Also we have

$$
x=\sum_{j=1}^{m} \sum_{i=1}^{n} \gamma_{i j} z_{i j}=\sum_{i=1}^{n} \sum_{j=1}^{m} \gamma_{i j} z_{i j}
$$

and

$$
\left(\sum_{j=1}^{m} \gamma_{i j}\right)^{-1} \sum_{j=1}^{m} \gamma_{i j} z_{i j} \in \text { face }\left(x_{i}\right)
$$

Since face $\left(x_{i}\right)$ is a split face, we have

$$
\lambda_{i} x_{i}=\sum_{j=1}^{m} \gamma_{i j} z_{i j} \text {. }
$$

Once more we use the fact that face $\left(x_{i}\right)$ is a split face and that

$$
\alpha_{j} y_{j}=\sum_{i=1}^{n} \gamma_{i j} z_{i j}
$$


to conclude that $\gamma_{i j}$ and $z_{i j}$ are uniquely determined. The proof is complete.

The next proposition was first proved by Wils (See Prop.3.20 in [22]). We will give a new proof based on Lemma 4.5. since the proposition has a corollary that is important in the subsequent work.

Proposition 4.6: Let $\mu, \nu \in \mathbb{M}_{X}$ and suppose $\mu$ is a central measure. Then $\mu$ and $\nu$ has a least upper bound in the choquet ordering $<$.

Proof: First we will suppose that $\mu$ and $\nu$ are discrete, i.e.

$$
\mu=\sum_{i=1}^{n} \lambda_{i} \epsilon_{x_{i}}, \nu=\sum_{j=1}^{m} \alpha_{j} \epsilon_{y_{j}}
$$

where all $\lambda_{i}, \alpha_{j}>0$. Let $\gamma_{i j}$ and $z_{i j}$ be as in Lemma 4.5 . and define

$$
\theta=\sum_{i, j} \gamma_{i j} \epsilon_{z_{i j}}
$$

Then we have $\mu<\theta$ and $\nu<\theta$.

Suppose $\pi \in \mathbb{M}_{\mathrm{X}}$ with $\mu<\pi$ and $\nu<\pi$. By Corollary I.3.3. in [3], there exist $\pi_{i}^{1}, \pi_{j}^{2} \in \mathbb{M}_{1}^{+}(K)$ such that

$$
\pi=\sum_{i=1}^{n} \lambda_{i} \pi_{i}^{1}, \pi_{i}^{1}>\epsilon_{x_{i}}
$$

and

$$
\pi=\sum_{j=1}^{m} \alpha_{j} \pi_{j}^{2}, \pi_{j}^{2}>\epsilon_{y_{j}}
$$

By Proposition II.3.3. in [3], there exist $\beta_{i j} \geq 0$ and $\pi_{1 j} \in \mathbb{M}_{1}^{+}(K)$ such that

$$
\lambda_{i} \pi_{i}^{1}=\sum_{j=1}^{m} \beta_{i j} \pi_{i j}
$$


and

$$
\alpha_{j} \pi_{j}^{2}=\sum_{i=1}^{n} \beta_{i j} \pi_{i j}
$$

Now we have

and

$$
\lambda_{i} r\left(\pi_{i}^{1}\right)=\lambda_{i} x_{i}=\sum_{j=1}^{m} \beta_{i j} r\left(\pi_{i j}\right)
$$

$$
\alpha_{j} r\left(\pi_{j}^{2}\right)=\alpha_{j} y_{j}=\sum_{i=1}^{n} \beta_{i j} r\left(\pi_{i j}\right)
$$

and by the uniqueness statement in Lemma 4.5 og we get $r\left(\pi_{i j}\right)=z_{i j}$ and $f_{i j}=\gamma_{i j}$. Thus we have $\theta<\pi$.

Now let $\mu, \nu \in \mathbb{M}_{X}$ be arbitrary with $\mu$ central. Let $\left\{\mu_{\alpha}\right\},\left\{\nu_{\beta}\right\}$ be nets of discrete measures in $\mathbb{M}_{X}$ such that $\nu_{\beta} \rightarrow \nu, \mu_{\alpha} \rightarrow \mu$, $\nu_{\beta}<\nu$ for all $\beta, \mu_{\alpha}<\mu$ for all $\alpha$ and such that $\mu_{\alpha}$ is central for all $\alpha$.

For all $\alpha$ and $\beta$, let $\theta_{\alpha \beta}$ be the least element in $\mathbb{M}_{X}$ such that $\mu_{\alpha}<\theta_{\alpha \beta}$ and $\nu_{\beta}<\theta_{\alpha \beta}$.

Let $\theta$ be a cluster point for $\left\{\theta_{\alpha \beta}\right\}$

If $\pi \in \mathbb{M}_{x}$ and $\mu<\pi, \nu<\pi$, then $\mu_{\alpha}<\pi$ and $\nu_{\beta}<\pi$ for all $\alpha, \beta$, and hence $\theta_{\alpha \beta}<\pi$ and $\theta<\pi$.

Let $f \in C(K)$ be a convex function and let $\epsilon>0$. There exist an $\alpha_{0}$ such that if $\alpha \geq \alpha_{0}$, then

$$
\mu_{\alpha}(f) \leq \mu(f) \leq \mu_{\alpha}(f)+\epsilon \text {. }
$$

Since for every $\beta, \mu_{\alpha}<\theta_{\alpha \beta}$, we have

$$
\mu(f) \leq \theta_{\alpha \beta}(f)+\epsilon, \quad \alpha \geq \alpha_{0}
$$

Hence

$$
\mu(f) \leq \theta(f)+\epsilon
$$

Since $\epsilon>0$ was arbitrary, $\mu<\theta$. In the same way we show that $\nu<\theta$. (In fact $\theta_{\alpha \beta}$ converges to $\theta$. ) The proof is complete. 
Corollary 4.7: Let $x \in K$ and let $\mu \in \mathbb{M}_{x}$ be a central measure. Then $\mu<\nu$ for all $\nu \in Q_{x}$.

Proposition 4.8: Let $\mu \in \mathbb{M}_{x}$ be a central measure. Then the set $\left\{\nu \in \mathbb{M}_{X}: \mu<\nu\right\}$ is a face of $\mathbb{M}_{X}$.

Proof: Suppose $\mu$ is discrete, i.e. $\mu=\sum_{i=1}^{n} \lambda_{i} \epsilon_{i}$ where $\lambda_{i}>0$ all $i$ and $\sum_{i=1}^{n} \lambda_{i}=1$.

Suppose $\nu=\lambda \nu_{1}+(1-\lambda) \nu_{2}>\mu$ where $\nu_{1}, \nu_{2} \in \mathbb{M}_{x}$ and $\lambda \in\langle 0,1\rangle$. Since $\mu<\nu$, we can find $\pi_{i} \in \mathbb{M}_{1}^{+}(K)$ such that $r\left(\pi_{i}\right)=x_{i}$ and $\nu=\sum_{i=1}^{n} \lambda_{i} \pi_{i}$ (see corollary I.3.3. in [3]). By Proposition II.3.3. in [3], there exist $\partial_{i j} \geq 0$ and $\pi_{i j} \in \mathbb{M}_{1}^{+}(K)$ such that

and

$$
\lambda_{i} \pi_{i}=\sum_{j=1}^{2} \partial_{i j} \pi_{i j} \quad, i=1, \ldots, n,
$$

$$
\lambda \nu_{1}=\sum_{i=1}^{n} \partial_{i 1} \pi_{i 1} \quad,(1-\lambda) \nu_{2}=\sum_{i=1}^{n} \partial_{i 2} \pi_{i 1}
$$

Since $\lambda \in\langle 0,1\rangle$, we have $\sum_{i=1}^{n} \partial_{i j} \neq 0$ for $j=1,2$.

If $\partial_{i j} \neq 0$, then $r\left(\pi_{i j}\right) \in$ face $\left(x_{i}\right)$. Since $\mu$ is central and

$$
\lambda r\left(\nu_{1}\right)=\lambda x=\sum_{i=1}^{n} \partial_{i 1} r\left(\pi_{i 1}\right)
$$

and

we have

$$
(1-\lambda) r\left(\nu_{2}\right)=(1-\lambda) x=\sum_{i=1}^{n} \partial_{i 2} r\left(\pi_{i 2}\right),
$$

$$
r\left(\pi_{i 1}\right)=r\left(\pi_{i 2}\right)=x_{i}
$$

and

$$
\partial_{i 1} \lambda^{-1}=\partial_{i 2}(1-\lambda)^{-1}=\lambda_{i}
$$


Hence $\epsilon_{x_{i}}<\pi_{i 1}, \pi_{i 2}$, and $\mu<\nu_{1}, \nu_{2}$. Thus $\left\{\nu \in \mathbb{M}_{x}: \mu<\nu\right\}$ is a face of $\mathbb{M}_{X}$.

Suppose next that $\mu$ is an arbitrary central measure in $\mathbb{N}_{X}$. Let $\left\{\mu_{\alpha}\right\}$ be a net in $\mathbb{M}_{X}$ of discrete central measures converging to $\mu$, and such that $\mu_{\alpha}<\mu$ for all $\alpha$. Then we have

$$
\left\{\nu \in \mathbb{M}_{x}: u<\nu\right\}=\underset{\alpha}{n}\left\{\nu \in \mathbb{M}_{x}: \mu_{\alpha}<\nu\right\} \text {. }
$$

Hence $\left\{\nu \in \mathbb{M}_{\mathbf{X}}: \mu<\nu\right\}$ is a face. The proof is complete.

\section{Corollary 4.9: Every central measure is simplicial.}

Proof: We use Proposition 4.8 and argue as in $(v) \Longrightarrow$ (i) of the proof of Theorem 1.5 .

Remark: We also have a more elementary proof of Corollary 4.9. It goes as follows: Suppose $\mu \in \mathbb{M}_{X}$ is central. Let $\mu_{1}, \mu_{2} \in \mathbb{M}_{X}$ and $\lambda \in<0,1>$ be such that $\mu=\lambda \mu_{1}+(1-\lambda) \mu_{2}$. By writing if necessary $\mu$ as

$$
\mu=\left(\frac{1}{2}+\frac{\lambda}{2}\right) \mu_{1}^{i}+\left(\frac{1}{2}-\frac{\lambda}{2}\right) \mu_{2}
$$

where

$$
\mu_{1}^{\prime}=\left(\frac{2 \lambda}{1+\lambda}\right) \mu_{1}+\left(\frac{1-\lambda}{1+\lambda}\right) \mu_{2}
$$

we can assume $\mu_{2} \ll \mu_{1}$.

Let $B \subseteq K$ be a Borel set. If $\mu_{1}(B)=0$, then $\mu_{2}(B)=0$, and if $\mu_{1}(B)=1$, then $\mu_{2}(B)=1$. If $\mu_{1}(B) \in\langle 0,1\rangle$, then an easy argument shows that $\mu_{1}(B)=\mu_{2}(B)$. Hence $\mu_{1}=\mu_{2}=\mu$, and $\mu$ is simplicial.

Definition: If a subset $S$ of $K$ is a union of faces, then we shall say that $S$ is a $\sigma$-face. 
Proposition 4.10: The subset of $\mathbb{M}_{1}^{+}(\mathrm{K})$ of all central measures is a $\sigma$-face.

Proof: Let $\mu_{1}, \mu_{2} \in \mathbb{M}_{1}^{+}(\mathbb{K})$ and let $\lambda \in\langle 0,1\rangle$ be such that $\mu=\lambda \mu_{1}+(i-\lambda) \mu_{2}$ is a central measure. We will show that $\mu_{1}$ is a central measure.

Let $B$ be a Borel subset of $K$ such that $\mu_{1}(B) \in\langle 0,1\rangle$. Then $\mu(B) \in\langle 0,1\rangle$. Hence

$$
r\left(\left.\mu(B)^{-1} \mu\right|_{B}\right) \circ r\left(\left.\mu(K B)^{-1} \mu\right|_{K-B}\right) \text {. }
$$

Since

$$
r\left(\left.\mu_{1}(B)^{-1} \mu_{1}\right|_{B}\right) \in \operatorname{face}\left(r\left(\left.\mu(B)^{-1}\right|_{B}\right)\right)
$$

and

$$
r\left(\left.\mu_{1}(K \cdot B)^{-1} \mu_{1}\right|_{K \cdot B}\right) \in \operatorname{face}\left(r\left(\left.\mu(K \cdot B)^{-1} \mu\right|_{K} B\right)\right)
$$

we have by Proposition 3.1. that

$$
r\left(\left.\mu_{1}(B)^{-1} \mu_{1}\right|_{B}\right) \circ r\left(\left.\mu_{1}(K \cdot B)^{-1} \mu_{1}\right|_{K-B}\right) \text {. }
$$

The proof is complete.

Definition: By a maximal central measure in $\mathbb{M}_{x}$ we shall mean a sentral measure that is maximal in Choquet's ordering among the central measures in $M_{X}$.

Remark: Wils has shown that every $\mathrm{x} \in \mathrm{K}$ can be represented by a unique maximal central measure. (See [20], [21], [22] and [3].) In [22; Prop.3.11] Wils showed that the subset of $\mathbb{M}_{1}^{+}(K)$ of all maximal central measures is a $\sigma$-face. Let $\mu \in \mathbb{M}_{1}^{+}(K)$. We shall write $\Lambda(\mu)$ for the set $\underline{\Lambda(\mu)}=\{B: B$ Borel set in $K$ and $\mu(B) \in<0,1\rangle\}$. 
Also for each $B \in \Lambda(\mu)$ we shall write:

$$
\begin{aligned}
& \underline{\underline{\lambda}}_{B}=\mu(B), \underline{\mu_{1 B}}=\left.\lambda_{B}^{-1} \mu\right|_{B}, \underline{\mu_{2 B}}=\left.\left(1-\lambda_{B}\right)^{-1} \mu\right|_{K \cdot B}, \\
& \underline{x}_{1 B}=r\left(\mu_{1 B}\right) \text { and } \underline{x_{2 B}}=r\left(\mu_{2 B}\right) . \text { Furthermore we shall }
\end{aligned}
$$

write $F(\mu)$ for the set

$$
\underline{F(\mu)}=\left\{\nu \in \mathbb{N}_{r(\mu)}: \mu<\nu\right\}
$$

Theorem 4.11: Let $x \in K$ and let $\mu \in \mathbb{M}_{X}$ be such that $\mu<\nu$ for all $\nu \in Q_{x}$. The following statements are equivalent:

(i) $u$ is a central measure.

(ii) face $\left(x_{1 B}\right) \cap$ face $\left(x_{2 B}\right)=\varnothing$ for all $B \in \Lambda(\mu)$

(iii) face $\left(F\left(\mu_{1 B}\right)\right) \cap$ face $\left(F\left(\mu_{2 B}\right)\right)=\varnothing$ for all $B \in \Lambda(\mu)$

(iv) If $B \in \Lambda(\mu)$ and $\sigma \in F\left(\mu_{1 B}\right)$ and $\lambda \in F\left(\mu_{2 B}\right)$, then $\sigma \perp \lambda$

Proof: (i) $\Rightarrow$ (ii) Follows from the definition of a central measure.

(ii) $\Rightarrow$ (i) Let $B \in \Lambda(\mu)$. We have to show that $\mathrm{x}_{1 \mathrm{~B}} \mathrm{x}_{2 \mathrm{~B}}$. By Iemma 4.1. and Iemma 4.2. it is enough to show that $x$ has a unique decomposition after face $\left(x_{1 B}\right)$ and face $\left(x_{2 B}\right)$.

Let $\alpha \in\langle 0,1\rangle$ and let $u_{i} \in$ face $\left(x_{i B}\right), i=1,2$, be such that

$$
x=\alpha u_{1}+(1-\alpha) u_{2}
$$

Let $\eta_{i} \in Q_{u_{i}}$. Then

$$
\nu=\alpha \eta_{1}+(1-\alpha) \eta_{2} \in Q_{x}
$$

Hence $\mu<v$.

By Corollary I.3.3. in [3] there exist $\tau_{i} \in Q_{x_{i B}}, i=1,2$, such that 


$$
\nu=\lambda_{B} \tau_{1}+\left(1-\lambda_{B}\right) \tau_{2},
$$

and $\mu_{i B}<\tau_{i}$ for $i=1,2$.

By Proposition II.3.3. in [3] there exist $\partial_{1}, \ldots, \partial_{4} \geq 0$ and $\pi_{1}, \ldots, \pi_{4} \in \mathbb{M}_{1}^{+}(K)$ such that

$$
\lambda_{B} \tau_{1}=\partial_{1} \pi_{1}+\partial_{2} \pi_{2},\left(1-\lambda_{B}\right) \tau_{2}=\partial_{3} \pi_{3}+\partial_{4} \pi_{4}
$$

and

$$
\alpha \eta_{1}=\partial_{1} \pi_{1}+\partial_{3} \pi_{3},(1-\alpha) \eta_{2}=\partial_{2} \pi_{2}+\partial_{4} \pi_{4} .
$$

If $\partial_{3} \neq 0$ then

$$
r\left(\pi_{3}\right) \in \text { face }\left(u_{1}\right) \cap \text { face }\left(x_{2 B}\right)=\varnothing .
$$

Hence $\partial_{3}=0$.

If $\partial_{2} \neq 0$, then

$$
r\left(\pi_{2}\right) \in \text { face }\left(u_{2}\right) \cap \text { face }\left(x_{1 B}\right) \neq \varnothing .
$$

Hence $\partial_{2}=0$.

Thus we have

$$
\lambda_{\mathrm{B}} \tau_{1}=\alpha \eta_{1}
$$

Hence $\lambda_{B}=\alpha$ and $u_{i}=x_{i B}, i=1,2$, and we have shown that $x$ has a unique decomposition after face $\left(x_{1 B}\right)$ and face $\left(x_{2 B}\right)$. (ii) $\Longrightarrow$ (iii) Follows from Iemma 2.1. and from a simple argument by contradiction.

(iii) $\Rightarrow$ (ii) Assume (iii) is fulfilled and that for some $B \in \Lambda(u)$, there is an $u \in$ face $\left(x_{1 B}\right) \cap$ face $\left(x_{2 B}\right)$.

Let $\sigma \in Q_{u}$, and let $\left.\lambda_{i} \in<0,1\right]$ and $u_{i} \in$ face $\left(x_{i B}\right)$ be such that

$$
x_{i B}=\lambda_{i} u+\left(1-\lambda_{i}\right) u_{i} \quad, i=1,2 .
$$

Let $\nu_{i} \in Q_{u_{i}}, i=1,2$. Then we have

$$
\lambda_{i} \sigma+\left(1-\lambda_{i}\right) \nu_{i} \in F\left(\mu_{i B}\right) \quad, i=1,2 .
$$


Hence $\sigma \in \bigcap_{i=1,2}$ face $\left(F\left(u_{i B}\right)\right)$.

We have obtained a contradiction. Hence face $\left(x_{1 B}\right) \cap$ face $\left(x_{2 B}\right)=\varnothing$. (iii) $\Rightarrow$ (iv) Follows from Lemma 2.1. (iv) $\Longrightarrow$ (iii) We have that

$$
\operatorname{face}(F(u))=U\{\text { face }(\nu): \nu \in F(\mu)\}
$$

(See [1]).

Let $B \in \Lambda(u)$ and suppose there is a $\nu \in$ face $\left(F\left(\mu_{1 B}\right)\right) n$ face $\left(F\left(\mu_{2 B}\right)\right)$. Then there exist $\sigma \in F\left(\mu_{1 B}\right)$ and $\lambda \in F\left(\mu_{2 B}\right)$ such that $y \in$ face $(\sigma) \cap$ face $(\lambda)$. By Proposition 1.2. and Lemma 2.1. we get that $\sigma \not \lambda \lambda$. This completes the proof.

Corollary 4.12: Let $\mu=\sum_{i=1}^{n} \lambda_{i} \epsilon_{x_{i}} \in \mathbb{M}_{x}\left(\operatorname{all} \lambda_{i}>0\right)$. The following statements are equivalent:

(i) $\mu$ is central

(ii) $\mu<\nu$ for all $\nu \in Q_{X}$, and face $\left(x_{i}\right) \cap$ face $\left(x_{j}\right)=\varnothing$ for $i \neq j$.

Proof: (i) $\Rightarrow$ (ii) is clear.

(ii) $\Longrightarrow$ (i) We argue as in the proof of Lemma 4.2. to show face $(x)=\operatorname{co}\left(\bigcup_{i=1}^{n}\right.$ face $\left.\left(x_{i}\right)\right)$. That each face $\left(x_{i}\right)$ is a split face in face $(x)$ is proved as $(i i) \Rightarrow(i)$ in Theorem 4.11. was proved. 
[1] Alfsen, E.M.: On the geometry of Choquet simplexes. Math. Scand. 15, 97-110 (1964).

[2] Alfsen, E.M.: On the decomposition of a Choquet simplex into a direct convex sum of complementary faces. Math. Scand. 17, 169-176 (1965).

[3] Alfsen, E.M.: Compact convex sets and boundary integrals. Ergebnisse der Nathematik, Springer Verlag, Germany (1971).

[4] Alfsen, E.M. and Bai Andersen, T.: Split faces of compact convex sets. Proc. Iondon Math. Soc. 21, 415-442 (1970).

[5] Alfsen, E.M. and Effros, E.: Structure in Real Banach Spaces. To Appear.

[6] Asimow, I. and Ellis, A.J.: Facial deoomposition of linearly compact simplexes and separation of functions on cones. Pacific Jour. of Math. 34, 301-309 (1970).

[7] Douglas, R.G.: On extremal measures and subspace density. Michigan Math. Jour. 11, 644-652 (1964).

[8] Edwards, D.A.: Separation de fonctions réelles definies sur un simplexe de Choquet. C.R.Acad.Sci. Paris 261, 2798-2800 (1965).

[9] Gamelin, T.W.: Uniform algebras. Prentice-Hall (1969).

[10] Hirsberg, B.: A measure theoretic characterization of paraliell - and split faces and their connection with function spaces and algebras. Aarhus Universitet, Various Publication Series, No 16 (1970).

[11] Køhn, J.: Barycenters of Unique Maximal Measures. Jour. of Funct. Analysis 6,76-82 (1970). 
[12] Lima, $\AA$ : On continuous convex functions and split faces. To appear in Proc. Iondon Math. Soc.

[13] Perdrizet, F.: Espaces de Banach ordonnés et idéaux. J. Math. pures et appl. 49, 61-98 (1970).

[14] Peressini, A.I.: Ordered topological vector spaces, New York: Harper \& Row (1967).

[15] Phelps, R.R.: Lectures on Choquet's theorem. Van Nostrand (1966).

[16] Riesz, F.: Sur quelques notions fondamentales dans la theorie generale des operations linéaires. Ann. of Math. 41, 174-206 (1940).

[17] Rogalski, M.: Caracterisation des simplexes par des proprietés portant sur les faces fermés et sur les ensembles compacts de points extremeaux. Math. Scand., 28, 159-181 (1971).

[18] Sakai, S.: On the central decomposition for positive functionals on $\mathscr{C}^{*}$-algebras. Trans. Amer. Math. Soc. 118, 406-419 (1965).

[19] Sakai, S.: $C^{*}$-Algebras and $W^{*}$-Algebras. Ergebnisse der Mathematik, Springer Verlag, Germany (1971).

[20] Wils, W.: Désintegration centrale des formes positives sur les $\mathscr{C}^{*}$-algebras. C.R. Acad. Sci. Paris 267, 810812 (1968).

[21] Wils, W.: Désintégration centrale dans une partie convexe compacte d'un espace localement convexe. C.R. Acad. Sci. Paris 269, 702-704 (1969).

[22] Wils, W.: The ideal center of partially ordered vector spaces. Acta Math. 127, 41-77 (1971). 